\title{
Interactive comment on "Process studies at the air-sea interface after atmospheric deposition in the Mediterranean Sea: objectives and strategy of the PEACETIME oceanographic campaign (May-June 2017)" by Cécile Guieu et al.
}

Anonymous Referee \#2

Received and published: 15 April 2020

The manuscript provides a description of the background, rationale, objectives, planning and execution of the 'PEACETIME' project investigating the influence of atmospheric dust deposition in the Mediterranean Sea. The manuscript provides potentially useful information to the community, both as a component of a special issue presenting the results of this study and hence a reference point for the other manuscripts, and as a case study in how to plan and execute studies of this type, which may be of use for others planning similar research projects in the future. However, I was left wondering Discussion paper whether the manuscript as stands provides the best introduction to the special issue 
as there is no attempt at providing synthesis or even a broad overview of the results of the study which will presumably be presented within the other manuscripts. If the authors currently plan to produce an additional overview/introductory manuscript then I would suggest they might consider merging these pieces of work, although it should be noted that the current manuscript is already quite long, in particular currently having 18 individual figures (see below).

Interactive

If the manuscript is to remain broadly in the current form I wondered whether the authors might broaden the potential interest to a wider community through adding in a section towards the end on 'lessons learnt' or similar. Namely, given the outlined plan and execution, are there recommendations the authors could make on how other groups might undertake and even improve on such a study in the future? Such a section might add value not least because the use of multiple real time observational and forecast data products described does indicate a level of adaptive planning of the cruise track which is perhaps beyond that usually performed. In the absence of some broader discussion of 'lesson learnt', as indicated above, I was personally left wondering whether the material in the manuscript might be considerably condensed and combined with a board overview of the results presented in the other special issue manuscripts.

Irrespective of whether a broader overview manuscript is planned, I note that there were a number of places where additional details might have been provided which were unlikely to compromise other publications. For example, how large was the observed rain deposition event (Line 475)? Related to this section, what does 'lowest most intense' (Line 481) mean?

Some additional general points:

It would be useful to have station locations indicated on more of the figures, in particular figures $7,8,9 \& 11$. It might also be worth compositing these figures as subpanels to enable easier comparison across them. Indeed I found I spent a lot of time moving

Printer-friendly version

Discussion paper 
backwards and forwards between different figures and the text and I think it would help readability if both the set of figures used and the formatting of these be reconsidered.

There were also a few places where referencing could have been improved. For example, the Okubo-Weiss parameter and Finite Size Lyapunov Exponents are not explained or referenced (Lines 408-409).

Minor corrections: There are a large number of minor typographic and/or grammatical errors throughout the manuscript. Some of these are listed (often as suggested alternative) below, but I will likely have missed many others and the whole manuscript requires a thorough proof read and edit.

Line 22: '. . .we provide a state of the art regarding..' state of the art review?

Line 25: rephrase

Line 32: 'in contrasted areas'

Line 51: 'in addition to these continuous'

Line 67: between less than 10\%?

Line 74 \& 74: '...also allow quantification of export below... for the marine biosphere...'

Line 124: 'water column'

Line 148: 'the probability of catching'

Line 165: 'associated with the rainy period'

Line 218: 'lead to changing the planned'

Line 236: 'the relevance of following the initial track was discussed in view of several'

Printer-friendly version

Line 253: sentence appears incomplete

Discussion paper

Line 283: maybe 'leading to the decision to start the' 
Line 332: 'every 15 minute'?

Line 398: '. . . strategy, with the aim of avoiding regions of. ..'

Line 409: 'exponent'

Line 420: 'the presence of a dust plume'

Line 436: 'On the 31st of May... between the islands of Sicily and Sardinia'

Line 446: 'On the 1st of June'

Line 454: during the first half of June 5th

Line 458: 'richer in'

Line 489: MVP is not defined here on first use

Line 526: '. . due to the rainfall. ..'

Line 528: needs rephrasing

Line 546: intermittent signals

Line 592: '. . . productivity and high probability of wet dust deposition.'

Line 1246: 'allowing detection of'

Interactive comment on Biogeosciences Discuss., https://doi.org/10.5194/bg-2020-44, 2020. 